\section{Zimbabwe's vaccination momentum needs an 'urgent boost' ahead of an imminent COVID-19 resurgence}

To the Editor: Since the first cases were recorded in March 2020, Zimbabwe has already experienced three distinct waves of the COVID-19 pandemic. The impact on the population, both direct and indirect, has been progressively more damaging with each wave. Emerging evidence from settings with high vaccination rates shows that these experienced a marked reduction in fatalities from COVID-19, alongside significant protection of vaccinated populations from severe morbidity. However, Zimbabwe remained with a high case fatality, close to $4 \%$, during the third wave which occurred from June to August 2021. ${ }^{[1]}$ During this period, despite being ahead of many other African countries, Zimbabwe had $<10 \%$ of its eligible population vaccinated, with population-wide benefits of vaccination not yet realisable as these would have required an estimated herd immunity threshold of $70-90 \%$.

Zimbabwe has approved five vaccines for use in the country, namely the Sinopharm, Sinovac, Covaxin, Sputnik V and Janssen vaccines, though the first two are the more readily available. As shown in Fig. 1, the initial pattern was of slow uptake, characterised by vaccine hesitancy among the targeted population of frontline healthcare workers. Subsequently, as vaccine availability and confidence improved, and the programme was extended to other eligible populations, there was an increase in uptake. This was followed by a marked surge in demand during the third wave, reportedly characterised by long queues, supply shortages and logistical challenges. As the third wave subsided, with a substantial decrease in deaths, the vaccination momentum slowed considerably. Zimbabwe's COVID-19 vaccination programme recorded a record high of 100 000 individuals a day during the third wave. The number fell to an average of 22000 a day during the first 3 weeks of October 2021, and it is estimated that $\sim 1$ million individuals have not yet received their second dose as scheduled. ${ }^{[1]}$ Over the past month the number of individuals who received at least one dose has increased marginally from $35 \%$ to the current $38 \%$.

As COVID-19 resurgence is expected in Zimbabwe, efforts to improve uptake are essential to protect the population from the damaging effects of COVID-19. This is particularly true in view of the country's strained healthcare sector, with markedly

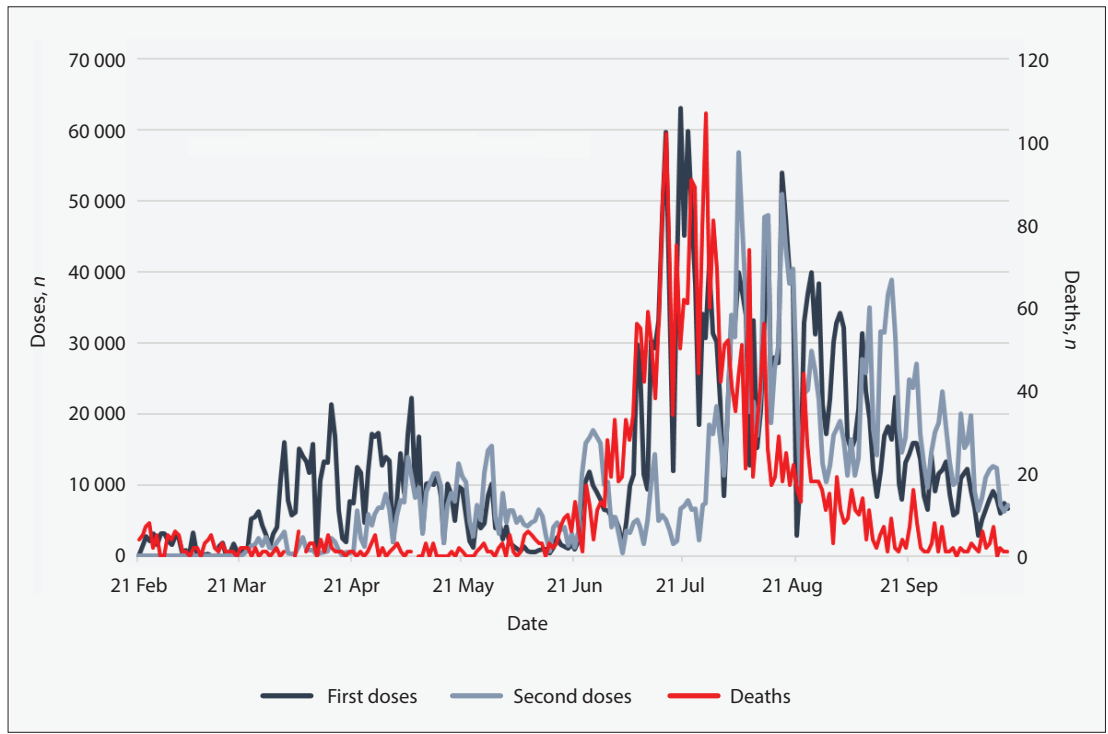

Fig. 1. Presentation for first and second vaccine doses and deaths in Zimbabwe, 21 February21 September 2021.

diminished capacity and resilience to deal with harsh surges in the burden of cases. An adequately vaccinated population is needed to protect both the health system and the population from the strain and adverse outcomes associated with severe COVID-19. Renewed strategies are therefore urgently required to restore and maintain the country's vaccination momentum, propelling it towards attaining its herd immunity threshold ahead of a further resurgence.

While it is not clear why the momentum is being lost, and it may be attributable in part to a belief that the worst of the pandemic is over, it is also a result of the continued circulation of falsehoods, myths and misconceptions around vaccine effectiveness, safety and the purpose of vaccination. The Ministry of Health and Child Care of Zimbabwe (MoHCC), through its risk communication and community engagement pillar, must therefore devise innovative strategies to make it clear to the population that COVID19 is still a threat, and that vaccines are safe and effective. Firstly, communication strategies that engage rather than coerce populations are needed for this purpose. These would involve understanding and adequately dealing with the genuine concerns of the target population. Secondly, resource mobilisation for urgent vaccine outreaches is important in order to bring vaccines to the communities where people live, overcoming barriers, particularly in marginalised areas with inadequate road networks and inaccessible to public transport. Thirdly, private doctors should be encouraged to enhance their efforts to administer COVID-19 vaccines in their practices, as this increases the number of available vaccination centres and decongests government clinics. Fourthly, advantage must be taken of existing community structures such as chiefs and councillors acting as health advocates, as they are trusted within their communities and can help in the drive to improve uptake. ${ }^{[2]}$ Persuasive ways of reaching the local apostolic religions with a theological objection to modern healthcare methods are required, especially as this population may also not adhere to control protocols such as avoiding large gatherings and physical distancing. ${ }^{[3]}$

The MoHCC and relevant stakeholders must distribute accessible safety and effectiveness data on vaccines to the population and aim for their countrywide dissemination in accessible formats through the diverse and available sources of social media, ensuring wide population reach. Additionally, attention should be paid to emerging concerns within the population around vaccines, in order to be able to engage with them effectively. These processes should be accompanied by mechanisms that ensure continuous supply chains and avoid stock ruptures, in a context where $\sim 35000$ people who received their first dose of Covaxin in May 2021 have not been able to receive a second dose owing to unavailability of the vaccine or knowledge of alternatives. ${ }^{[4]}$ Moreover, the development of guidelines for specific populations such as pregnant women and adolescents must be expedited to avoid rampant confusion among both service providers and prospective vaccine recipients. ${ }^{[5]}$

In conclusion, the government of Zimbabwe, through the MoHCC and relevant public health stakeholders, must urgently 
find innovative ways of rejuvenating the COVID-19 vaccination momentum ahead of further inevitable waves of the pandemic. This search must be prioritised to make sure that the country makes reasonable progress towards reaching the herd immunity threshold, realising the population-wide benefits of widespread vaccination, and protecting the people from the devastating health and socioeconomic consequences of COVID-19.

\section{Grant Murewanhema}

Unit of Obstetrics and Gynaecology, Department of Primary Health Care Sciences, Faculty of Medicine and Health Sciences, University of Zimbabwe, Harare, Zimbabwe

\section{Tafadzwa Dzinamarira}

School of Health Systems and Public Health, University of Pretoria, South Africa; ICAP at Columbia University, Harare, Zimbabwe anthonydzina@gmail.com

Munyaradzi Mapingure, Innocent Chingombe, Solomon Mukwenha, Godfrey Musuka

ICAP at Columbia University, Harare, Zimbabwe

\section{Roda Madziva}

School of Sociology and Social Policy, University of Nottingham, UK

\section{Helena Herrera}

School of Pharmacy and Biomedical Sciences, University of

Portsmouth, UK

1. Ministry of Health and Child Care of Zimbabwe (MoHCC). COVID-19 situational reports. http:// www mohcc. gov.zw/index. php?option $=c o m$ phocadownload \&view $=$ category\&id $=158$ \&ttemid $=741$ (accessed 23 October 2021)

2. Dzinamarira T, Musuka G. When culture, traditions and public health clash: A paradigm shift urgently needed to stem the spread of COVID-19 in Zimbabwe. S Afr Med J 2021;111(4):279. https://doi.org/10.7196/SAMJ.2021.v111i4.15637

3. Dzinamarira T, Nachipo B, Phiri B, Musuka G. COVID-19 vaccine roll-out in South Africa and Zimbabwe: Urgent need to address community preparedness, fears and hesitancy. Vaccines 2021;9(3):250. https://doi.org/10.3390/vaccines 9030250

4. Moyo T. India vaccines recipients to restart the process. The Chronicle, 18 October 2021. https:// www.chronicle.co.zw/india-vaccines-recipients-to-restart-process/ (accessed 23 October 2021).

5. Murewanhema G, Dzinamarira T, Herrera H, Musuka G. COVID-19 vaccination for pregnant women in Zimbabwe: A public health challenge that needs an urgent discourse. Public Health Pract (Oxf) 2021;2:100200. https://doi.org/10.1016/j.puhip.2021.100200

S Afr Med J 2021;111(12):1164-1165. https://doi.org/10.7196/SAMJ.2021.v111i12.16231 